\title{
Affect recognition as an independent social function determinant in schizophrenia
}

\author{
Yi-Ju Pan ${ }^{\mathrm{a}}$, Sue-Huei Chen ${ }^{\mathrm{a}, \mathrm{b}}$, Wei J. Chen ${ }^{\mathrm{a}, \mathrm{c}}$, Shi-Kai Liu ${ }^{\mathrm{a}, \mathrm{d}, *}$ \\ ${ }^{a}$ Department of Psychiatry, National Taiwan University Hospital, Taipei, Taiwan \\ ${ }^{\mathrm{b}}$ Department of Psychology, National Taiwan University, Taipei, Taiwan \\ ${ }^{\mathrm{c}}$ Institute of Epidemiology, College of Public Health, National Taiwan University, Taipei, Taiwan \\ ${ }^{\mathrm{d}}$ Department of Psychiatry, Far Eastern Memorial Hospital, Taipei, Taiwan
}

\begin{abstract}
Introduction: Facial affect recognition deficits may represent specific deficits and contribute to social dysfunction in patients with schizophrenia. Whether their impacts on social dysfunction are independent to those caused by deficits in basic neurocognition and clinical symptoms needs to be further delineated.

Method: Association patterns between affect recognition and basic neurocognitive abilities in 40 acute and 33 stable patients with schizophrenia were compared to explore whether their interrelationships changed across clinical stages. The independent contribution of affect recognition deficits to social dysfunction was explored by multivariate models controlling for general intellectual ability, basic neurocognition, and clinical symptoms.

Results: Affect recognition deficits were associated with social role performances, self-care, and contributed independently to global social functioning in stable patients but not in acute patients. Conversely, affect recognition deficits were associated with impaired basic neurocognitions in acute patients but not in stable patients.

Conclusion: In stabilized community patients with schizophrenia, affect recognition deficits were relatively independent to basic neurocognition and had significant social functional consequences.
\end{abstract}

(C) 2009 Published by Elsevier Inc.

\section{Introduction}

Facial affect recognition deficit has been extensively documented in patients with schizophrenia [1-5]. Failure to accurately perceive the affective expressions of others is likely to impair subsequent emotional processing and result in inadequate deciphering of complex dynamic emotional cues. Deficits in recognition of facial affects may thus compromise successful social context integration and interfere with acquisition of social skills [6]. Indeed, cross-sectional studies demonstrated that facial affect recognition deficits in patients with schizophrenia were associated with diminished functioning in general and

* Corresponding author. Department of Psychiatry, Far Eastern Memorial Hospital, Ban-Ciao City, Taipei 22060, Taiwan. Tel.: +886 2 8966 7000x2936; fax: +886289665567.

E-mail addresses: formosa@mail.femh.org.tw, formosaotto@gmail.com (S.-K. Liu). specific social domains, including interpersonal relationships, communication, occupational performances, and personal care [7-9]. Findings from longitudinal studies further confirmed that such associations were robust over time and affect recognition deficits predicted outcomes in work functioning/independent living [10], social, and community functioning [11].

Despite such plausible causal links, however, the specificity and extent of contribution of facial affect recognition deficits to social dysfunction remain to be further defined [12], given that there are still concerns whether facial affect recognition deficits represent specific deficits with unique social function consequences or they arise from the generalized neurocognitive impairment frequently observed in patients with schizophrenia [1315]. Also, because affect recognition deficits are intricately entwined with negative symptoms [16], another important determinant for social functional outcomes [17], the specific contribution of affect recognition deficits to social 
dysfunction needs be delineated from those caused by basic neurocogntion and negative symptoms.

Neurophysiologically, facial affect recognition can be a distinct neural event, given that abnormal cerebral activations during processing of facial affects in patients with schizophrenia have been shown to be specifically over areas implicated in the autonomic processing of emotions, for example, the limbic and paralimbic regions [18-20]. Nevertheless, disconcerted interactions between activities of emotion-related basic limbic structures and executionbased medial frontal regions have also been implicated for causing emotion processing deficits in schizophrenia $[21,22]$. Such independence/interdependence between emotional perception and neurocognition is thus of theoretical importance because it attests to the basic distinction between their underlying neural substrates and mechanisms [23,24].

Experimental evidence suggests that affect recognition deficits in patients with schizophrenia may result from the flow-on effects of a generalized deficit in early visual processing [20], as well as perturbed controlled processing of facial emotions [25]. Specifically, there are deficits in structural encoding of facial details that hampered the initial automatic iconic readout [26]; and further downstream, emotional information processing may as well be affected by deficits in executive function [27,28], selective attention, and sustained attention [29] because these neurocognitive processes were demonstrated to be moderately associated with emotional perception deficits and reallife social function impairments in patients with schizophrenia [11,30-32].

However, findings regarding whether affect recognition and basic neurocognition are differentially involved in patients with schizophrenia are still inconsistent across different disease stages. Patients with schizophrenia in acute stage were reported to fail both affect recognition and facial recognition tasks nondifferentially, and deficits in facial affect recognition were associated with impairments in Continuous Performance Test (CPT) and Span of Apprehension Test (SPAN), a relationship persisting up to 3 months after an acute exacerbation [5]. Yet, there are contrary evidence suggesting that after controlling for performances in the general perception tasks, affect recognitions deficits manifested as specific deficits in patients in acute stage of schizophrenia [14]. Likewise, although patients with chronic schizophrenia were found to specifically fail the affect recognition tasks [18,33-35], a study on patients under chronic extended care, a population frequently manifested advanced neurocognitive impairments, suggested that affect recognition deficits were part of a generalized neurocognitive deficit [14]. Moreover, in treatment-resistant schizophrenia, the performances in emotional perception were shown to link specifically to the early visual scanning processes and sustained attention [2].

Such discrepant findings might be partly attributable to the diversities in the characteristics of study samples $[11,14]$ and the nature of the emotional tasks used [15]because individuals in remission may perform better than individuals in an acute phase of schizophrenia in emotional recognition tasks [36] and deficits in emotional perception may correlate with severity of disease [8] and increased illness duration $[4,9]$. Furthermore, patients with schizophrenia vary widely in severity and profile of neurocognitive deficits, which might also be related to disease stage [37] and entail different developmental trajectories [38]. The issue is complicated further that acute and chronic patients with schizophrenia are often different in their severity of negative symptoms. Because affect recognition deficits are intricately entwined with clinical negative symptoms [16], negative symptoms may have modified the relationship between affect recognition and social functioning, probably through disturbances in the regulation of emotion [23].

In this regard, current study aimed to disentangle such complex interactions among affect recognition, basic neurocognition, and clinical symptoms, hence to clarify the interrelationships between affect recognition deficits and basic neurocognition and explore whether facial affect recognition deficits contributed independently to social dysfunction in patients with schizophrenia. We included patients recently recovering from acute episodes, as well as those stable community-dwelling patients to demonstrate possible disease stage-related effects on their interrelationships.

\section{Materials and methods}

\subsection{Subjects}

Subjects in current study were recruited from the acute inpatient ward and day-care rehabilitation center of the Department of Psychiatry, National Taiwan University Hospital (NTUH), a university-affiliated teaching hospital providing both acute inpatient care and day rehabilitation programs for severe mental disorders.

Forty acute remitting inpatients with schizophrenia and 33 stable community-dwelling outpatients with schizophrenia were recruited. The diagnosis of schizophrenia was made and confirmed by 2 board-certificated psychiatrists using the semistructured Diagnostic Interview for Genetic StudyChinese Version [39,40]. Subjects with the following conditions were excluded: history of mental retardation, pervasive developmental disorder, traumatic brain injury with consciousness loss, epilepsy, electroconvulsive therapy, and alcohol and/or other psychoactive substance dependence/ abuse during previous 1 year. The inpatient treatment of an acute episode of schizophrenia at NTUH typically lasts for 4 to 6 weeks and the mean (SD) duration of admission of the acute inpatients is 33.8 (10.0) days. In NTUH, patients were regularly referred to rehabilitation programs after clinical condition stabilized, which typically started around 1 to 2 weeks after admission. Patients would make try-out visits within 1 week before planned discharge to evaluate their adjustments in the community and plan for further rehabilitation programs. Accordingly, acute remitting inpatients were 
evaluated within the 1 week of their planned discharges when certain degree of social/occupational functioning had been restored. The stable outpatients with schizophrenia were recruited from the regular attendants of the day care program at NTUH. They must have attended the programs for at least 3 months and were clinically stable with a Clinical Global Impressions (CGI) - Severity score less than 3.

To provide an estimation of the degree of impairments in neurocognitive performances, 40 healthy comparison subjects were recruited through advertisements in the hospital. The comparison subjects were also screened by Diagnostic Interview for Genetic Study-Chinese version to exclude concurrent Axis I psychiatric disorders and Axis II schizophrenic spectrum personality disorders. Other exclusion criteria were the same as those for patients with schizophrenia. The study protocol had been approved by the ethical review committee of NTUH, and all subjects participated after giving their written informed consents.

\subsection{Assessments}

\subsubsection{Clinical symptom measures}

The Positive and Negative Syndrome SchedulesChinese version (PANSS-CH) was used by board-certificated psychiatrists (YJP and SKL) well experienced in PANSS-CH to evaluate the severity of clinical symptoms. Specifically, factor analytic study demonstrated that the 14 positive and negative subscale items of the PANSS-CH regrouped into 4 symptom dimensions, that is, negative, disorganization, delusion/hallucination, and excitement, which had been demonstrated to be more closely associated with underlying neurocognitive processes [41]. The 14 PANSS-CH items hence were selected to represent the core schizophrenic psychopathology, and mean symptom dimension scores were calculated by the summed average of the item scorings included in each dimension.

\subsubsection{Social function assessment}

The Personal and Social Performance Scale (PSP) is developed and validated after the Diagnostic and Statistical Manual of Mental Disorders, Fourth Edition, Social and Occupational Functional Assessment Scale to evaluate social functioning by using objective information obtained in patients' real-life settings [42]. Based on patient's best possible performing levels during previous 1 month, PSP is purported to provide a composite estimate for global social functioning from 4 separate social functional domains, including (1) social role performances (PSP_SR): socially useful activities, including work and study; (2) interpersonal relationships (PSP_IP): personal and social relationships; (3) self-care (PSP_SC); and (4) disturbing and aggressive behaviors (PSP_AG). Each functional domain is rated independently along a 6-point Likert scale (0, absence; 1 , mild; 2, manifest; 3 , marked; 4, severe; and 5, very severe), according to the description for each anchoring point. Higher scores indicate poorer performances. A global social functioning score of PSP (PSP_GL) could then be generated from the 4 individual PSP functional domain ratings following algorithm provided by PSP. The scorings of PSP_GL range from 0 to 100 , paralleling and reflecting those Social and Occupational Functional Assessment Scale functioning levels. A higher PSP_GL score indicates better global functioning.

Personal and Social Performance Scale assessments were performed by senior psychiatrists in charge of the care of the patients after standard training sessions and reliability exercises in an independent sample of 16 schizophrenic patients. The guidelines provided by PSP were used for the raters to gather all relevant observations from staffs in direct care of the patients to rate the 4 PSP functional domains. The intra-class correlation coefficients for PSP_SR, PSP_IP, PSP_SC, PSP_AG, and PSP_GL were 0.96, 0.96, 0.92, 0.82, and 0.98 , respectively, indicating good interrater reliability. Because social function of the acute inpatients might be underestimated due to restricted milieu exposure and active psychotic symptoms, the ratings of PSP social functional domains were based on the information obtained in the very 1 week before planned discharge, during which patients had partially remitted and were undertaking rehabilitation assessments and programs, as well as try-outs to the community with reencountering with families and relatives. The day care patients had been regularly attending the rehabilitation programs for at least 3 months, during which they were participating in a variety of occupational and social training programs, and their social and occupational performances had been systemically evaluated every 1 month by the rehabilitation team.

\subsubsection{Neuropsychologic assessments}

2.2.3.1. Facial affect recognition. The computerized Taiwanese version of the facial expression subtest of The Diagnostic Analysis of Non-Verbal Accuracy II (DANVA-2-TW) [43] was used to assess the accuracy for recognition of the 4 basic human emotions, for example, happiness, sadness, anger, and fearfulness. Although the original DANVA-2 has been applied to clinical populations including chronic schizophrenia [7], schizotypy [44], and pediatric anxiety [45] in American society, the exclusive use of photos of western races and English language limits its use in Taiwan. In this regard, the DANVA-2-TW has been developed as a parallel version of the original DANVA-2 and is composed of photos of Han faces and Mandarin to simulate the scenarios in Taiwanese society. The DANVA-2TW has been demonstrated to have good reliability and applied to patients with schizophrenia, their first-degree relatives, and normal comparison subjects with good discriminating validity [43]. The facial expression subtest of DANVA-2-TW consisted of 24 gray-scaled posed emotional photos, 6 for each basic emotion, which are selected from a pool of 120 photos that have been previewed by 60 healthy subjects and attain at least $80 \%$ agreement 
rates about which emotion category they belong to. Each photo is presented on a 15-in laptop computer screen for 500 milliseconds. Subjects are asked to make a choice of the photos' emotional categories among the 4 basic emotions. The available response time was 5 seconds. The duration for stimuli display and available response time were deliberately made brief to impose a time constraint to restrict excessive cognitive reappraisal. The performance index is the percentage of correct categorization.

\subsubsection{Selective attention. The computerized SPAN [46]} is a measure of early visual processing and iconic readout. Subjects are asked to determine whether target numbers appear among an array of numbers on the computer screen and respond by pressing a button. Three sets of stimuli were used, consisting of 3-, 6-, and 12-number arrays, respectively. The stimuli lasted for 100 milliseconds and the interstimulus interval (ISI) was 1400 milliseconds. A total of 64 stimuli were used for each session. The performance index was the percentage of correct response.

\subsubsection{Visual-spatial working memory. The visual-spatial} $\mathrm{N}$-back task is designed as a visual-spatial counterpart of the verbal N-back test for evaluating the capacity of visualspatial processing. As in the verbal N-back test, successive stimuli appear on the computer screen with a fixed ISI. Instead of the digits, the stimuli in the visual-spatial N-back are figures exhibiting a pattern of 4 square blocks aligned in line and one among which is shaded. Although the pattern is fixed and remains the same across all stimuli, the position of the shaded block changes from stimulus to stimulus. The visual-spatial N-back task requires that subjects respond by pressing the keyboard when the position of the shaded block in the current stimulus corresponds to that in the $n$th stimulus back. For example, in 1-back condition, subjects respond only when the position of the shaded block in the current figure is the same as that in the figure immediately preceding current one. In 0-back condition, subjects have to respond whenever the stimuli appear on the screen. There were 4 different N-back sessions, for example, the 0-back, 1-back, 2-back, and 3-back conditions. The stimuli lasted for 100 milliseconds with available response time of 1400 milliseconds, and the next stimulus would appear 300 milliseconds after subjects made the response. There are 64 stimuli for each N-back session, and the performance index is the correct rate.

2.2.3.4. Sustained attention. Sustained attention was assessed with the CPT, version 2.20, operated on a Sunrise Systems machine (Pembroke, Mass). The procedure and its test-retest reliability have been described in detail elsewhere [47]. Briefly, numbers from 0 to 9 were randomly presented for 50 milliseconds each, at a rate of 1 per second. Subjects undertook the undergraded 1-9 CPT task, in which they were asked to respond whenever a 9 preceded by 1 appeared on the screen. A total of 331 trials, $34(10 \%)$ of which were target stimuli, were presented over 5 minutes for each session. The sensitivity index $d^{\prime}$ was derived from the hit rate (probability of response to target trials) and false-alarm rate (probability of response to nontarget trials) [48], which measures an individual's ability to discriminate the target stimuli from a nontarget stimuli.

2.2.3.5. Trail making tasks. In the Trail Making Test, pseudorandomly placed circles with numbers (Trail Making A), and with both numbers and letters (Trail Making B) have to be connected in a line as fast as possible in a fixed order [49]. Besides the psychomotor speed hypothesized to be assessed by Trail Making A, the Trail Making B additionally assesses subjects' ability to shift mental sets. The difference in time (seconds) for completing Trail Making B and A (Trail Making B_A) hence is used as an index for mental flexibility.

\subsection{Statistical analysis}

Group comparisons were performed using $\chi^{2}$ test (for categorical variables) and analysis of variance with post hoc Bonferroni comparisons (for continuous variables). Associations between affect recognition, basic neurocognitve tasks, and general intellectual ability were explored by correlational analyses (Pearson $r$ ). Relationships between PSP scores, clinical symptoms, and neurocognitive measures were examined by nonparametric correlational analyses (Spearman $\rho$ ). The correlational analyses were performed on comparison subjects, acute remitting patients, and stable patients separately to examine whether different groups showed distinct association patterns. Multivariate regression analysis was further used to explore the relative contributions of demographic characteristic, basic neurocognition, affect recognition, and clinical symptoms to social functions. Owing to the ordinal nature and narrow ranges of distribution of the PSP domain scores, regression analysis was only performed on PSP_GL scores. In preparation of the multivariate regression analysis, scores of PSP_GL were logtransformed to meet the normality requirements. Considering that there were many independent variables of interest and extensive correlations between demographic variables, neurocognitive performances, and clinical symptoms, we selected the variables to be included in final regression model through the following steps. First, variables were initially screened by their first-order correlations with social function scores. Second, collinearity diagnoses were performed while regressing PSP_GL on the preliminary set of selected independent variables, including demographic characteristics (age, education), neurocognitive measures (affect recognition, CPT, N-Back, SPAN, Wechsler Adult Intelligence Scale-Revised, Veral Intelligence Quotient (VIQ) and Performance Intelligence Quotient (PIQ)), and clinical symptoms (negative symptoms and disorganization symptoms). Variables with insignificant regression coefficients and those with variance inflating factor larger than 2.0, 
Table 1

Demographic characteristics and neurocognitive performances in patients with schizophrenia and comparison subjects

\begin{tabular}{|c|c|c|c|}
\hline & $\begin{array}{l}\text { Acute } \\
\text { schizophrenia } \\
(n=40)\end{array}$ & $\begin{array}{l}\text { Stable } \\
\text { schizophrenia } \\
(\mathrm{n}=33)\end{array}$ & $\begin{array}{l}\text { Comparisons } \\
(\mathrm{n}=40)\end{array}$ \\
\hline n $(\%)$ of male & $20(50)$ & $\begin{array}{c}18(54.5) \\
\text { Mean (SD) }\end{array}$ & $15(37.50)$ \\
\hline Age (y) & $30.60(7.43)$ & $32.76(8.14)$ & $33.75(10.64)$ \\
\hline Education (y) & $13.58(2.75)$ & $13.79(2.25)$ & $14.43(2.67)$ \\
\hline WAIS-R full $\mathrm{IQ}^{\mathrm{a}}$ & 87.28 (12.63) & $95.91(13.79)$ & $113.75(14.84)$ \\
\hline \multicolumn{4}{|l|}{$\begin{array}{l}\text { Neurocognitive } \\
\text { measures }\end{array}$} \\
\hline $\begin{array}{l}\text { Affect } \\
\text { recognition } \\
{(\text { correct/total })^{\mathrm{a}}}^{\text {a }}\end{array}$ & $0.54(0.20)$ & $0.68(0.11)$ & $0.73(0.11)$ \\
\hline $\begin{array}{l}\text { N_2 Back } \\
\text { (correct/total) }^{\mathrm{a}}\end{array}$ & $0.32(0.25)$ & $0.48(0.28)$ & $0.56(0.24)$ \\
\hline $\begin{array}{l}\text { SPAN_12 } \\
\text { (correct/total) }^{\mathrm{b}}\end{array}$ & $0.61(0.20)$ & $0.70(0.14)$ & $0.73(0.11)$ \\
\hline $\begin{array}{l}\text { CPT } d^{\prime} \text { raw } \\
\text { scores }^{\mathrm{b}}\end{array}$ & $3.18(0.96)$ & $4.21(0.79)$ & $4.45(0.51)$ \\
\hline $\begin{array}{l}\text { Trail Making } \\
\text { B_A }(\mathrm{sec})^{\mathrm{a}}\end{array}$ & $77.00(47.24)$ & $50.39(41.17)$ & $37.80(25.72)$ \\
\hline \multicolumn{4}{|l|}{$\begin{array}{l}\text { Disease } \\
\quad \text { associated } \\
\text { characteristics }\end{array}$} \\
\hline Age at onset & $24.50(8.31)$ & $21.97(6.30)$ & N/A \\
\hline $\begin{array}{c}\text { Duration of } \\
\text { illness }(y)^{\mathrm{c}}\end{array}$ & $6.38(4.94)$ & $10.72(6.64)$ & N/A \\
\hline $\begin{array}{l}\text { No. of previous } \\
\text { admissions }\end{array}$ & $2.42(1.68)$ & 1.45 (1.75) & N/A \\
\hline $\begin{array}{l}\text { Chlorpromazine } \\
\text { equivalents } \\
(\mathrm{mg})\end{array}$ & $448.75(228.22)$ & $453.94(232.74)$ & N/A \\
\hline \multicolumn{4}{|c|}{ Function assessments } \\
\hline PSP_SR ${ }^{c}$ & $2.95(0.90)$ & $1.97(1.43)$ & N/A \\
\hline PSP_IP ${ }^{c}$ & $2.42(1.04)$ & $1.55(1.23)$ & N/A \\
\hline PSP_SC ${ }^{c}$ & $0.90(0.97)$ & $0.48(0.62)$ & N/A \\
\hline PSP $\_A G^{c}$ & $0.60(0.98)$ & $0.06(0.24)$ & N/A \\
\hline PSP_GL & $49.72(14.55)$ & $62.88(17.97)$ & N/A \\
\hline $\begin{array}{l}\text { PANSS total } \\
\text { scores }^{\mathrm{c}}\end{array}$ & $64.53(12.60)$ & $48.24(12.68)$ & N/A \\
\hline \multicolumn{4}{|c|}{ PANSS symptom dimensions } \\
\hline Negative & $1.75(1.00)$ & $1.73(0.94)$ & N/A \\
\hline $\begin{array}{l}\text { Hallucination/ } \\
\text { delusion }^{c}\end{array}$ & $3.14(0.82)$ & $1.64(0.67)$ & N/A \\
\hline Disorganization & $2.13(0.96)$ & $1.71(0.86)$ & N/A \\
\hline Excitement $^{\mathrm{c}}$ & $1.63(0.68)$ & $1.06(0.24)$ & N/A \\
\hline $\mathrm{CGI}^{\mathrm{c}}$ & $3.28(1.07)$ & $1.15(1.00)$ & N/A \\
\hline
\end{tabular}

ANOVA indicates analysis of variance; WAIS-R full IQ, Wechsler Adult Intelligence Scale-Revised, Full Intelligence Quotient; N_2 Back, N_2_Back Test; SPAN_12, Span of Apprehension Test 12 arrays.

a ANOVA with post hoc Bonferroni comparisons: comparison subjects $>$ Stable schizophrenia $>$ acute schizophrenia $(P<.05)$.

b ANOVA with post hoc Bonferroni comparisons: comparison subjects $=$ stable schizophrenia $>$ acute schizophrenia $(P<.05)$.

${ }^{\mathrm{c}}$ Independent-sample $t$ test $(P<.005)$.

indicating significant collinearity problems, were dropped from subsequent regressions. However, to retain the basic theoretical distinctions between those categories of independent variables that might have independent contributions to social function, some neurocognitive variables that did not show significant association were retained; for example, SPAN measures were retained to control for early visual processing. The final regression analyses were then performed by regressing PSP_GL on the selected independent variables. This same set of independent variables were applied to both acute remitting and stable patients with schizophrenia to test whether factors contributing to social functioning differed between these 2 patient groups. Data from comparison subjects were meant for descriptive contrasts with patients with schizophrenia; they hence were not included in the regression analyses. The statistical significance was set at a $P$ value of less than .05. All statistical analyses were done using SPSS version 10.0 (SPSS, Chicago, IL).

\section{Results}

Patients with schizophrenia did not differ significantly from comparison subjects in demographic characteristics (Table 1). However, they had poorer performances in all neurocognitive measures. Among patients with schizophrenia, acute remitting patients were comparable to stable community patients in age, male-to-female ratio, years of formal education, and general intellectual abilities (Table 1). At the time of assessments, the acute remitting patients had partially recovered and had mild to moderate clinical symptoms as evidence by their mean (SD) CGI score of 3.28 (1.07). In contrast, the community patients did not exhibit overt symptoms with a mean (SD) CGI score of 1.15(1.00). The acute patients had shorter duration of illness, more previous inpatient admissions, higher PANSS total scores, and lower mean PSP global and domain scores (Table 1). Dosage of antipsychotics did not differ between these 2 patient groups. For most neurocognitive tasks, stable community patients performed better than acute remitting patients and were comparable to comparison subjects in selective attention, sustained attention, and trail making tasks (Table 1).

Table 2

Correlations between affect recognition and neurocognitive measures in patients with schizophrenia and comparison subjects ${ }^{\mathrm{a}}$

\begin{tabular}{lccc}
\hline & $\begin{array}{l}\text { Acute remitting } \\
\text { schizophrenia } \\
(\mathrm{n}=40)\end{array}$ & $\begin{array}{l}\text { Stable community } \\
\text { schizophrenia } \\
(\mathrm{n}=33)\end{array}$ & $\begin{array}{l}\text { Comparison } \\
\text { subjects } \\
(\mathrm{n}=40)\end{array}$ \\
\hline & \multicolumn{3}{c}{$\begin{array}{c}\text { Affect } \\
\text { recognition }(\% \text { correct })\end{array}$} \\
N_2 Back & $0.48^{* *}$ & 0.08 & $0.36^{*}$ \\
$\quad$ (correct/total) & & & \\
SPAN 12 (correct/total) & $0.32^{*}$ & 0.08 & 0.07 \\
CPT d & 0.13 & -0.03 & 0.24 \\
Trail making B_A (s) & -0.31 & -0.24 & 0.08 \\
FIQ & $0.52^{* *}$ & 0.17 & 0.27 \\
\hline
\end{tabular}

FIQ, Wechsler Adult Intelligence Scale-Revised Full Intelligence Quotient.

\footnotetext{
${ }^{a}$ Pearson correlation coefficients.

* $P<.05$.
}

** $P<.005$. 
Table 3

Differential association patterns in PSP ratings, clinical symptoms, and neurocognitive performances in patients with schizophrenia ${ }^{\text {a }}$

\begin{tabular}{|c|c|c|c|c|c|c|c|c|c|c|}
\hline & $\begin{array}{l}\text { Negative } \\
\text { symptoms }\end{array}$ & $\begin{array}{l}\text { Hallucination/ } \\
\text { delusion }\end{array}$ & Disorganization & Excitement & FIQ & $\begin{array}{l}\text { Affect } \\
\text { recognition }\end{array}$ & N_2 Back & SPAN_12 arrays & CPT $\_d^{\prime}$ & Trail B_A \\
\hline \multicolumn{11}{|c|}{ Acute patients with schizophrenia $(n=40)$} \\
\hline PSP_SR & -0.14 & $0.35^{*}$ & 0.06 & $0.35^{*}$ & -0.14 & -0.09 & -0.07 & -0.01 & -0.28 & -0.03 \\
\hline PSP_IP & -0.03 & 0.26 & 0.19 & $0.36^{*}$ & -0.24 & -0.15 & -0.22 & -0.13 & -0.28 & -0.01 \\
\hline PSP_SC & $0.28^{*}$ & -0.12 & $0.32 *$ & 0.10 & -0.28 & -0.26 & -0.21 & -0.11 & -0.04 & 0.13 \\
\hline PSP_AG & 0.02 & 0.20 & 0.20 & $0.35^{*}$ & 0.04 & -0.19 & -0.17 & -0.28 & -0.12 & -0.23 \\
\hline PSP_GL & -0.04 & -0.27 & -0.15 & $-0.44 * *$ & 0.23 & 0.14 & 0.15 & 0.11 & 0.27 & -0.10 \\
\hline \multicolumn{11}{|c|}{ Stable patients with schizophrenia $(n=33)$} \\
\hline PSP_SR & $0.69 * * *$ & $0.44^{*}$ & $0.65 * * *$ & -0.11 & $-0.63 * * *$ & $-0.43^{*}$ & $-0.48^{* *}$ & -0.23 & $-0.32^{*}$ & 0.14 \\
\hline PSP_IP & $0.68 * * *$ & $0.51^{* *}$ & $0.62 * * *$ & -0.12 & $-0.58 * * *$ & -0.29 & $-0.51 * *$ & -0.22 & -0.22 & -0.10 \\
\hline PSP_SC & $0.60 * * *$ & 0.27 & $0.52 * * *$ & 0.01 & $-0.55^{* * *}$ & $-0.40 *$ & -0.15 & 0.07 & -0.22 & -0.02 \\
\hline PSP_GL & $-0.73 * * *$ & $-0.47 * *$ & $-0.65 * * *$ & 0.14 & $0.60 * * *$ & $0.35^{*}$ & $0.50^{* *}$ & 0.21 & $0.29 *$ & -0.07 \\
\hline
\end{tabular}

${ }^{\text {a }}$ Nonparametric correlation by Spearman $\rho$.

$* P<.05$.

** $P<.005$.

*** $P<.001$

Patterns of association between affect recognition, basic neurocognitive measures, and general intellectual ability were dissimilar among comparison subjects, acute remitting, and stable schizophrenic patients (Table 2). In healthy subjects, affect recognition was associated with visual-spatial working memory only. In acute remitting patients, affect recognition had extensive associations with the neurocognitive measures, including general intellectual ability, visual-spatial working memory, and selective attention. In sharp contrast, for stable community patients, affect recognition was not associated with general intellectual ability and the basic neurocognitive measures.

The association patterns between PSP scores and clinical/ neurocognitive measures were distinct for acute remitting patients and stable patients as well (Table 3). In acute remitting patients, PSP scores did not correlate with any neurocognitive measure and clinical symptoms, except the

Table 4

Factors contributing to global social functioning by multiple regression analyses in stable community schizophrenic patients

\begin{tabular}{lll}
\hline Models $^{\text {a }}$ & $\begin{array}{l}\text { Standardized regression } \\
\text { coefficient }(\beta)\end{array}$ & $P$ \\
\hline Negative symptom & -.456 & .001 \\
N_2 Back (\% correct) & .316 & .016 \\
SPAN_12 (\% correct) & -.109 & .431 \\
Facial affect recognition & .342 & .007 \\
CPT $d^{\prime}$ & .379 & .008 \\
Education (years) & -.346 & .03 \\
VIQ & .435 & .003 \\
\hline
\end{tabular}

VIQ indicates Wechsler Adult Intelligence Scale-Revised, Veral Intelligence Quotient.

${ }^{a}$ Model $R^{2}$ (adjusted) $=0.658, \mathrm{~F}=9.78, P<.0001$. Dependent variables: log-transformed PSP_GL score; independent variables: negative symptom factor, facial affect recognition, visual-spatial working memory (N_2 Back), selective attention (SPAN-12), sustained attention (CPT $\left.d^{\prime}\right)$, VIQ, and education. excitement symptoms. In contrast, for stable patients, PSP scores were extensively associated with Wechsler Adult Intelligence Scale-Revised, Full Intelligence Quotient (absolute values of $r=0.55-0.63$ ), affect recognition (absolute values of $r=0.35-0.43$ ), working memory (absolute values of $r=0.48-0.51$ ), CPT sensitivity index (absolute values of $r=0.29-0.32$ ) and clinical symptom dimensions, especially negative symptoms (absolute values of $r=0.60-0.73$ ) (Table 3).

For stable community outpatients, multivariate regression analyses on global social functioning revealed that negative symptoms, visual-spatial working memory, facial affect recognition, sustained attention, VIQ, and education were significantly associated with PSP global social functioning scores (Table 4). The model was highly significant with an adjusted $R^{2}$ of $0.66(\mathrm{~F}=9.78, P<.0001)$. When applied to acute remitting patients, the same model was not significant and global social functioning was not accounted for by any of the neurocognitive or clinical variables.

\section{Discussion}

Two important observations arose from current study. First, acute remitting and stabilized patients with schizophrenia had dissimilar association patterns between affect recognition and neurocognition. In acute remitting patients, affect recognition deficits were associated with impairments in selective attention, visual-spatial working memory, and general intellectual ability; whereas in stable communitydwelling patients, deficits in affect recognition were not associated with basic neurocognitive performances. Such findings illustrate the independence/interdependence between emotional perception and neurocognition across disease stages and suggest that facial affect recognition deficits may be related to decrements in general cognitive capacity in acute patients but relatively independent to basic 
neurocognition in stable patients. Second, in stable community patients, deficits in affect recognition were associated with functioning in various social functioning domains and contributed independently to impairment in global social functioning after controlling for education, basic neurocognitive performances, general intellectual ability, and clinical symptoms. Conversely, no such association was found in acute remitting patients. The findings help to disentangle the complex interactions between emotional perception, neurocognition, negative symptoms, and social dysfunction in schizophrenia.

Consistent with the general trend, the acute remitting patients performed significantly worse than stabilized community patients in affect recognition as well as in other basic neurocognitive abilities [36]. Also, significant associations were found between affect recognition and neurocognition. Neurophysiologically, basic neurocognition can be impeded substantially by the disruptive effects on attention and visual imagery with sudden increase in medication use during acute phase of schizophrenia [50], which may further compromise the already diminished visual scanning capacity, a salient feature of patients with schizophrenia [51], and interfere with the speed and accuracy in the automatic scanning processes. Failure to allocate visual attention and fixate on the salient facial features then ensues [50]. Deficits in basic neurocognitions hence can lead to impairments in judging emotional categories and valences and account for the associations between affect recognition and basic neurocognition.

Dissimilar to previous findings from community samples $[8,10,11]$, no association between affect recognition and basic neurocognition is found in current stabilized community patients. It is notable that stabilized community patients in current study performed close to normal comparison subjects in both affect recognition and basic neurocognition. Especially, their performances in SPAN and CPT were not impaired. It is then possible that the relative severity of impairments in those basic neurocognitive processes may be crucial in accounting for such discrepancies because basic neurocognitive processes, especially those pertaining to early visual processing and attention, set the preparatory stage for subsequent facial feature encoding and affect decoding [52], and spatial perception has been observed to be the best predictor of facial emotion identification [53]. When impairments in basic neurocognition are severe as to hamper the initial stage of facial affect processing, it will have cascading impacts on the downstream processing and result in deficits in facial affect recognition. Significant association between affect recognition basic neurocognition and a profile of generalized impairment hence will emerge. Conversely, in those who do not have overt impairment in basic neurocognition, affect recognition deficits, if there were any, will be unconnected with basic neurocognition and appear as specific deficits. In the same vein, it can be further asserted that, as long as the impairments in basic neurocognition are severe enough to interfere with the early visual processing, no matter if due to the impacts of an acute psychotic episode or the results of a long-term deterioration in the chronic course of schizophrenia, it will impact the performances in facial affect recognition. Findings from the current study indeed concur with such contention because the acute remitting patients had significant impairments in basic neurocognition, and affect recognition were related to a general neurocognitive impairment. However, it is still possible that fundamental changes in underlying neural circuits and mechanisms regulating emotion perception and neurocognition across disease stages have led to such hierarchical changes in their interrelationships, which remains to be proved by longitudinal studies directly measuring within-subject changes in neurophysiologic activities of relevant limbic-cortical circuits across disease stages of schizophrenia.

Another important finding from current study is that affect recognition deficits were associated with social role performance and self-care and contributed independently to impairments in global social functioning. Although moderate in magnitude, such associations are remarkable given that experimental measures of affect recognition in present study are limited to the early phase of facial affect discriminating and labeling, which are conceptually and practically remote to the complex real-world macrosocial functioning as assessed by the PSP. Although social dysfunction in schizophrenia has been postulated to reflect social cognition deficits, arising from patient's limitation in judging the meaning of perceived emotional cues rather than failure in affect recognition [54], current results nevertheless indicate that deficits in earlier emotional cue detecting stages are pivotal. Affect recognition deficits in the earlier stages may have direct impacts on the downstream deciphering and contextual understanding of the meaning of emotional cues, hence resulting on the impairments of the related social functioning [23]. Moreover, as suggested by various statistical models, facial affect recognition can indirectly affect macrosphere social functioning through their mediating or modulating effects on neurocognition [11,55-58].

Nonsocial neurocognitive performances in the present study significantly accounted for the variances in social functioning besides affect recognition and clinical symptoms. The findings replicate the results from numerous previous studies, which underscore the importance of basic neurocognitions and general intellectual ability in supporting abilities and skills needed for social functioning and community living [59]. Visual-spatial working memory emerged as the most significant predictor among the basic neurocognition in our regression model. Working memory has been considered as the building block for effective social functioning [60] and demonstrated to be key determinant for acquiring social behavioral skills in patients with schizophrenia [61]. Deficits in working memory can be postulated to constraint subject's capacity for acquisition of social/occupational skills [59]. Furthermore, working memory has been demonstrated to have enduring impacts on social functioning because it was 
predictive of social relationships and recreational activities even after 7 years [17].

The impacts of working memory deficits, however, should be further disentangled from those of negative symptoms [17] because both negative symptoms and working memory deficits have been demonstrated to be key determinants of functional outcome in schizophrenia [62]. Previous studies indicated that effects of negative symptoms on social functioning often diminished in strength after taking into account neurocognitive performances [63], and there were concerns about the conceptual overlaps between social function measures and negative symptoms because negative symptoms encompassed decreased social interaction, social interests, and affective expressions. In addition, the associations between affect recognition deficits and negative symptoms might be corollary to specific disturbances in the regulation of emotion because they were only found between affect recognition and those negative symptoms pertaining to emotional perception and expression, such as anhedonia or apathy [64]. Nevertheless, present results support that negative symptoms can have independent and additive contributions to social dysfunctions $[12,65]$.

Models about the interrelationships among emotional perception, basic neurocognition, general cognitive ability, and clinical symptoms can provide conceptual framework for understanding the pathways to social dysfunction in schizophrenia [11,32]. By circumscribing the range and nature of the functional consequences of affect recognition deficits, it is possible to target the core problems to facilitate optimal strategies for neurocognitive rehabilitation $[66,67]$ or psychopharmacologic interventions [68] specifically targeted at affect recognition [69]. Preliminary evidence suggested that such pharmacologic/neurocognitive interventions could improve neurocognitive functions in general [70] and affect recognition in specific [71]. For example, monetary reinforcement as well as promoting facial feedback via mimicry has been used with positive results [66]. However, whether such therapeutic gain can be sustained and generalized to different target population remains unclear. Further evaluations of the combined intervention strategies are warranted.

Several limitations should be kept in mind while interpreting the results. First, in acute inpatients, although social functioning was rated when they were relatively stabilized and receiving occupational and social rehabilitation, it may still be underestimated because they did not have the chance to fully participate in family and community activities for adequate social function ratings. Also, the impacts of residual psychotic symptoms might have masked those caused by cognitive deficits. Second, the neurocognitive assessments used in present study were restricted to those hypothetically related to early stage of emotional processing, which might have left out important neurocognitive processes in the entirety of the complex emotional processing. Third, although PSP represents an ecologically relevant measure of social functioning based on observations under real life situations, it nevertheless limits the generalizability of findings to other patient groups under different social settings. Because the stable community patients in the current study are relatively high-functioning, their functional achievements are limited in range and the applicability of the results to those in the early phase of the disease or those stabilized but chronically instituted populations remains to be further examined.

\section{Acknowledgment}

This research was supported by a grant from National Science Council of Taiwan (NSC 93-2314-B-002-251).

\section{References}

[1] Kohler CG, Bilker W, Hagendoorn M, Gur RE, Gur RC. Emotion recognition deficit in schizophrenia: association with symptomatology and cognition. Biol Psychiatry 2000;48:127-36.

[2] Kee K, Kern R, Green M. Perception of emotion and neurocognitive functioning in schizophrenia: what's the link? Psychiatr Res 1998;81 57-65.

[3] Sachs G, Steger-Wuchse D, Kryspin-Exner I, Gur RC, Katschnig H. Facial recognition deficits and cognition in schizophrenia. Schizophr Res 2004;68:27-35.

[4] Kucharska-Pietura K, David AS, Masiak M, Phillips ML. Perception of facial and vocal affect by people with schizophrenia in early and late stages of illness. Br J Psychiatry 2005;187:523-8.

[5] Addington J, Addington D. Facial affect recognition and information processing in schizophrenia and bipolar disorder. Schizophr Res 1998; 32:171-81.

[6] Green MJ, Waldron JH, Coltheart M. Emotional context processing is impaired in schizophrenia. Cognit Neuropsychiatry 2007;12: 259-80.

[7] Hooker C, Park S. Emotion processing and its relationship to social functioning in schizophrenia patients. Psychiatr Res 2002;112: 41-50.

[8] Poole JH, Tobias FC, Vinogradov S. The functional relevance of affect recognition errors in schizophrenia. J Internat Neuropsychol Soc 2000; 6:649-58

[9] Mueser KT, Doonan R, Penn DL, Blanchard JJ, Bellack AS, Nishith P, et al. Emotion recognition and social competence in chronic schizophrenia. J Abnorm Psychol 1996;105:271-5.

[10] Kee K, Green MF, Mintz J, Brekke JS. Is emotion processing a predictor of functional outcome in schizophrenia? Schizophr Bull 2003;29:487-97.

[11] Addington J, Saeedi H, Addington D. Facial affect recognition: a mediator between cognitive and social functioning in psychosis? Schizophr Res 2006;85:142-50.

[12] Dickinson D, Coursey RD. Independence and overlap among neurocognitive correlates of community functioning in schizophrenia. Schizophr Res 2002;56:161-70.

[13] Nelson AL, Combs DR, Penn DL, Basso MR. Subtypes of social perception deficits in schizophrenia. Schizophr Res 2007;94:139-47.

[14] Penn D, Combs D, Ritchie M, Francis J, Cassisi J, Morris S, et al. Emotion recognition in schizophrenia: further investigation of generalized versus specific deficit models. J Abnorm Psychol 2000;109:512-6.

[15] Johnston PJ, Devir H, Karayanidis F. Facial emotion processing in schizophrenia: no evidence for a deficit specific to negative emotions in a differential deficit design. Psychiatry Res 2006;143: 51-61. 
[16] Sergi MJ, Rassovsky Y, Widmark C, Reist C, Erhart S, Braff DL, et al Social cognition in schizophrenia: relationships with neurocognition and negative symptoms. Schizophr Res 2007;90:316-24.

[17] Milev P, Ho B, Arndt S, Andreasen N. Predictive values of neurocognition and negative symptoms on functional outcome in schizophrenia: a longitudinal first-episode study with 7-year followup. Am J Psychiatry 2005;162:495-506.

[18] Gur RE, McGrath C, Chan RM, Schroeder L, Turner T, Turetsky BI, et al. An fMRI study of facial emotion processing in patients with schizophrenia. Am J Psychiatry 2002;159:1992-9.

[19] Fakra E, Salgado-Pineda P, Delaveau P, Hariri AR, Blin O. Neural bases of different cognitive strategies for facial affect processing in schizophrenia. Schizophr Res 2008;100:191-205.

[20] Johnston PJ, Stojanov W, Devir H, Schall U. Functional MRI of facial emotion recognition deficits in schizophrenia and their electrophysiological correlates. Eur J Neurosci 2005;22:1221-32.

[21] Hempel A, Hempel E, Schonknecht P, Stippich C, Schroder J. Impairment in basal limbic function in schizophrenia during affect recognition. Psychiatr Res 2003;122:115-24.

[22] Streit M, Wolwer W, Gaebel W. Facial-affect recognition and visual scanning behaviour in the course of schizophrenia. Schizophr Res 1997;24:311-7.

[23] Abdi Z, Sharma T. Social cognition and its neural correlates in schizophrenia and autism. CNS Spectr 2004;9:335-43.

[24] Pinkham AE, Penn DL, Perkins DO, Lieberman J. Implications for the neural basis of social cognition for the study of schizophrenia. Am J Psychiatry 2003;160:815-24

[25] Van't Wout M, van Dijke A, Aleman A, Kessels RP, Pijpers W, Kahn RS. Fearful faces in schizophrenia: the relationship between patient characteristics and facial affect recognition. J Nerv Ment Dis 2007; 195:758-64.

[26] Turetsky BI, Kohler CG, Indersmitten T, Bhati MT, Charbonnier D, Gur RC. Facial emotion recognition in schizophrenia: when and why does it go awry? Schizophr Res 2007;94:253-63.

[27] Bozikas V, Kosmidis M, Anezoulaki D, Giannakou M, Karavatos A. Relationship of affect recognition with psychopathology and cognitive performance in schizophrenia. J Internat Neuropsychol Soc 2004;10: 549-58.

[28] Bryson G, Bell M, Lysaker P. Affect recognition in schizophrenia: a function of global impairment or a specific cognitive deficit. Psychiatr Res 1997;71:105-13.

[29] Combs DR, Gouvier WD. The role of attention in affect perception: an examination of Mirsky's four factor model of attention in chronic schizophrenia. Schizophr Bull 2004;30:727-38.

[30] Addington J, Addington D. Neurocognitive and social functioning in schizophrenia. Schizophr Bull 1999;25:173-82.

[31] Addington J, Addington D. Neurocognitive and social functioning in schizophrenia: a 2.5 year follow-up study. Schizophr Res 2000;44 47-56.

[32] Nienow TM, Docherty NM, Cohen AS, Dinzeo TJ. Attentional dysfunction, social perception, and social competence: what is the nature of the relationship? J Abnorm Psychol 2006;115:408-17.

[33] Heimberg C, Gur RE, Erwin RJ, Shtasel DL, et al. Facial emotion discrimination: III. Behavioral findings in schizophrenia. Psychiatr Res 1992;42:253-65.

[34] Borod JC, Martin CC, Alpert M, Brozgold A, et al. Perception of facial emotion in schizophrenic and right brain-damaged patients. J Nerv Ment Dis 1993;181:494-502.

[35] Walker E, McGuire M, Bettes B. Recognition and identification of facial stimuli by schizophrenics and patients with affective disorders. Br J Clin Psychology 1983;23:37-44.

[36] Penn DL, Sanna LJ, Roberts DL. Social cognition in schizophrenia: an overview. Schizophr Bull 2008;34:408-11.

[37] Liu SK, Hsieh MH, Huang TJ, Liu CM, Liu CC, Hua MS, et al. Patterns and clinical correlates of neuropsychologic deficits in patients with schizophrenia. J Formos Med Assoc 2006;105: 978-91.
[38] Liu SK, Hsieh MH, Hwang TJ, Hwu HG, Liao SC, Lin SH, et al. Reexamining sustained attention deficits as vulnerability indicators for schizophrenia: stability in the long term course. J Psychiatr Res 2006; 40:613-21.

[39] Chang CJ, Chen WJ, Liu SK, Cheng JJ, Yang WC, Chang HJ, et al. Morbidity risk of psychiatric disorders among the first degree relatives of schizophrenia patients in Taiwan. Schizophr Bull 2002; 28:379-92.

[40] Nurnberger Jr JI, Blehar MC, Kaufmann CA, York-Cooler C, Simpson $\mathrm{SG}$, Harkavy-Friedman J, et al. Diagnostic interview for genetic studies. Rationale, unique features, and training. NIMH Genetics Initiative. Arch Gen Psychiatry 1994;51:849-59 [discussion 863-844].

[41] Liu SK, Hwu HG, Chen WJ. Clinical symptom dimensions and deficits on the Continuous Performance Test in schizophrenia. Schizophr Res 1997:25:211-9.

[42] Morosini PL, Magliano L, Brambilla L, Ugolini S, Pioli R. Development, reliability and acceptability of a new version of the DSM-IV Social and Occupational Functioning Assessment Scale (SOFAS) to assess routine social functioning. Acta Psychiatr Scand 2000;101:323-9.

[43] Tseng HH. Nonverbal emotional perception and schizotaxic tendency: a comparative study of schizophrenic patients, their families, and the normal control. Master Thesis, Department of Psyhology, National Taiwan University 2003.

[44] Shean G, Bell E, Cameron CD. Recognition of nonverbal affect and schizotypy. J Psychol 2007;141:281-91.

[45] Easter J, McClure EB, Monk CS, Dhanani M, Hodgdon H, Leibenluft E, et al. Emotion recognition deficits in pediatric anxiety disorders: implications for amygdala research. J Child Adolesc Psychopharmacol 2005;15:563-70.

[46] Asarnow RF, Granholm E, Sherman T. Span of apprehension in schizophrenia. In: Steinhauer SR, Gruzelier JH, et al, editors. Neuropsychology, psychophysiology, and information processing. Handbook of schizophrenia, vol. 5. New York, NY: Elsevier Science; 1991. p. 335-70.

[47] Chen WJ, Hsiao CK, Hsiao LL, Hwu HG. Performance of the Continuous Performance Test among community samples. Schizophr Bull 1998;24:163-74.

[48] Swets JA. The relative operating characteristic in psychology: A technique for isolating effects of response bias finds wide use in the study of perception and cognition. Science 1973;182:990-1001.

[49] Reitan RM, Wolfson D. The Halstead-Reitan neuropsychological test battery. Tuscon: Neuropsychological Press; 1985.

[50] Williams LM, Loughland CM, Gordon E, Davidson D. Visual scanpaths in schizophrenia: is there a deficit in face recognition? Schizophr Res 1999;40:189-99.

[51] Whittaker JF, Deakin JF, Tomenson B. Face processing in schizophrenia: defining the deficit. Psychol Medicine 2001;31:499-507.

[52] Wynn JK, Lee J, Horan WP, Green MF. Using event related potentials to explore stages of facial affect recognition deficits in schizophrenia. Schizophr Bull 2008;34:679-87.

[53] Chan CC, Wong R, Wang K, Lee TM. Emotion recognition in Chinese people with schizophrenia. Psychiatry Res 2008;157:67-76.

[54] Brune M. Emotion recognition, "theory of mind," and social behavior in schizophrenia. Psychiatr Res 2005;133:135-47.

[55] Sergi MJ, Rassovsky Y, Nuechterlein KH, Green MF. Social perception as a mediator of the influence of early visual processing on functional status in schizophrenia. Am J Psychiatry 2006;163:448-54.

[56] Brekke JS, Nakagami E, Kee KS, Green MF. Cross-ethnic differences in perception of emotion in schizophrenia. Schizophr Res 2005;77: 289-98.

[57] Vauth R, Rusch N, Wirtz M, Corrigan P. Does social cognition influence the relation between neurocognitive deficits and vocational functioning in schizophrenia? Psychiatr Res 2004;128:155-65.

[58] Bell M, Tsang HW, Greig TC, Bryson GJ. Neurocognition, social cognition, perceived social discomfort, and vocational outcomes in schizophrenia. Schizophr Bull 2008 [electronic publication]. 
[59] Green MF, Kern RS, Heaton RK. Longitudinal studies of cognition and functional outcome in schizophrenia: implications for MATRICS. Schizophr Res 2004;72:41-51.

[60] Penn DL, Mueser KT, Spaulding W, Hope DA, Reed D. Information processing and social competence in chronic schizophrenia. Schizophr Bull 1995;21:269-81.

[61] Smith T, Hull J, Huppert J, Silverstein S. Recovery from psychosis in schizophrenia and schizoaffective disorder: symptoms and neurocognitive rate-limiters for the development of social behavior skills. Schizophr Res 2002;55:229-37.

[62] Winterer G, Coppola R, Goldberg T, Egan M, Jones D, Sanchez C, et al. Prefrontal broadband noise, working memory, and genetic risk for schizophrenia. Am J Psychiatry 2004;161:490-500.

[63] Velligan D, Bow-Thomas C, Mahurin R, Miller A, Halgunseth L. Do specific neurocognitive deficits predict specific domains of community function in schizophrenia? J Nerv Ment Dis 2000;188: 518-24.

[64] Suslow T, Roestel C, Arolt V. Affective priming in schizophrenia with and without affective negative symptoms. Eur Arch Psychiatry Clin Neurosci 2003;253:292-300.
[65] Revheim N, Schechter I, Kim D, Silipo G, Allingham B, Butler P, et al. Neurocognitive and symptom correlates of daily problem-solving skills in schizophrenia. Schizophr Res 2006;83:237-45.

[66] Penn DL, Combs D. Modification of affect perception deficits in schizophrenia. Schizophr Res 2000;46:217-29.

[67] Greenwood K, Landau S, Wykes T. Negative symptoms and specific cognitive impairments as combined targets for improved functional outcome within cognitive remediation therapy. Schizophr Bull 2005; 31:910-21.

[68] McGurk SR, Carter C, Goldman R, Green MF, Marder SR, Xie H, et al. The effects of clozapine and risperidone on spatial working memory in schizophrenia. Am J Psychiatry 2005;162:1013-6.

[69] Pinkham AE, Gur RE, Gur RC. Affect recognition deficits in schizophrenia: neural substrates and psychopharmacological implications. Expert Rev Neurother 2007;7:807-16.

[70] Barnett JH, Salmond CH, Jones PB, Sahakian BJ. Cognitive reserve in neuropsychiatry. Psychol Med 2006;36:1053-64.

[71] Herbener E, Hill S, Marvin R, Sweeney J. Effects of antipsychotic treatment on emotion perception deficits in first-episode schizophrenia. Am J Psychiatry 2005;162:1746-8. 